\section{Study of the Formation, Secretion and Trafficking of Membrane Vesicles Loaded with Polyurethane Nanoparti- cles in Staphylococcus aureus via Electron Microscopy}

\section{Roberta Curia ${ }^{1,2, *}$, Alessandro Erega ${ }^{1,2}$ and Marziale Milani ${ }^{1}$}

${ }^{1}$ Department of Materials Science, University of Milano-Bicocca, Milan, Italy

${ }^{2}$ Department of Biotechnology and Biosciences, University of Milano-Bicocca, Milano, Italy

\begin{abstract}
Staphylococcus aureus is a gram-positive bacterium permanently present in the oral cavity, able to colonize polyurethane dental prostheses and to form biofilm. The bacterial action on the polymeric material results in the biodestruction of the polyurethane and in the formation of micro- and nano-particles. Electron microscopy, having a key role in the investigation of nanoparticles-bacteria interactions, points out that bacteria internalize polyurethane nanoparticles enveloped in membrane vesicles. In this work we discuss the uptake process of nanoparticles and the role of the bacterial cytoskeleton, important for the vesicular trafficking. We show images of membrane vesicles loaded with one or more polyurethane nanoparticles inside the bacterial cell, astride the membrane and out of the cell, in the extracellular medium.

This work opens new ways to the nanomedicine in the comprehension of bacterial secretion of membrane vesicles and their interactions with host cells, and raises new issues about the nanoparticles delivery guided by the dynamics of bacterial infections, and the related toxicological risks.

Keywords: Electron microscopy; Infection; Membrane vesicles; Nanomedicine; Nanoparticles; Staphylococcus aureus; Toxicology
\end{abstract}

\section{Abbreviations}

FIB/SEM: Focused Ion Beam/Scanning Electron Microscope MV: $\quad$ Membrane Vesicle

*Corresponding author: Roberta Curia, Department of Biotechnology and Biosciences, University of Milano-Bicocca, Piazza della Scienza 2, 20125 Milan, Italy, Tel: +39 0264485175; E-mail: r.curia@campus.unimib.it

Citation: Curia R, Erega A, Milani M (2015) Study of the Formation, Secretion and Trafficking of Membrane Vesicles Loaded with Polyurethane Nanoparticles in Staphylococcus aureus via Electron Microscopy. J Nanotechnol Nanomed Nanobiotechnol 2: 005.

Received: April 21, 2015; Accepted: June 18, 2015; Published: July 07, 2015
NP: Nanoparticle

OMV: Outer Membrane Vesicle

PU: Polyurethane

STEM: Scanning Transmission Electron Microscope

TEM: Transmission Electron Microscope

\section{Short Communication}

In the last decade great interest has been demonstrated towards nanoparticles (NPs) especially for their unique characteristics which make NPs a powerful tool to work with in several fields, from engineering to medicine, from biology to materials sciences, from environmental sciences to (nano) toxicology [1]. In literature studies take into account different categories of NPs (metallic and non-metallic NPs, inert and reactive NPs); most of the papers talk about engineered NPs absorbed by inhalation, ingestion, via dermal penetration or intravenous perfusion [1]. The reason why NPs are widely used in so many fields of application is that they have a high surface/volume ratio, depending on the species they may be highly reactive, have unique physical and chemical properties, are able to easily pass through biological membranes, and finally they can be used for drug delivery [2,3]. All these characteristics on one hand make NPs useful, on the other hand NPs can turn out to be risky and harmful to handle, raising important issues about their toxicity, bioaccumulation and NPs-induced pathologies [4-6].

In this work we focus on a new type of NP, a non-engineered one derived from the biodestruction of polyurethane (PU) dental prostheses carried out by Staphylococcus aureus. In vitro experiments together with a "correlative" FIB/SEM/(S)TEM (Focused Ion Beam/Scanning Electron Microscope/(Scanning) Transmission Electron Microscope) analysis show that bacteria are able to adhere to the polymeric material (24-48 hours), form microcolonies ( 48 hours), and after longer incubation time (7 days) they are able to form biofilm [7]. Biofilm maturation is accompanied by the appearance of an exopolysaccharide (EPS) matrix. Bacteria embedded in biofilm operate a corrosive action on the plastic surface, becoming the PU a feeding source for the microorganisms $[8,9]$, and as a result the PU surface results seriously damaged $[7,10,11]$.

PU samples with different types of surfaces were provided by Dentalur Russia, Russian Federation. The culture of $S$. aureus was isolated from a patient with a periodontal disease and was incubated with PU slices of different roughness. A PU slice with a non-sawed surface in broth and a broth with S. aureus with no PU were the control samples. Biological specimens prepared for transmission electron microscopy underwent primary fixation, washing, secondary fixation, infiltration with transition solvent and resin, embedding, curing and cutting. Since traditional drying operations led to structural changes in the sample, the standard dehydration processes were not applied. Samples were stained with Alcian Blue in order to preserve the exopolysaccharide (EPS) matrix and coated with copper sulphate [7].

TEM and STEM images were acquired with a tecnai F20 X-TWIN microscope (FEI Company, USA) equipped with a $200 \mathrm{kV}$ FEG column and a CCD detector. Bright Field (BF), Dark Field (DF) and High Angle Annular Dark Field (HAADF) techniques have been used 
to obtain images with improved contrast and resolution, in order to gain more information about PU NPs' size and their relative position compared to cell structures [7].

FIB/SEM images visible in Didenko et al. [7] document that as a consequence of the biodestructive action of $S$. aureus, micro- and nano-particles (which size ranges from $2-3 \mathrm{~nm}$ to $10 \mathrm{~nm}$ ) detach themselves from the bulk $\mathrm{PU}$ and are embedded in the exopolysaccharide (EPS) matrix. Through TEM and STEM images in Didenko et al. [10] and Curia et al. [11] it is possible to detect PU NPs within S. aureus.

Electron microscopy images show the actual internalization of PU NPs inside bacterial cells, but which are the uptake mechanisms that induce NPs to enter cells? NPs are able to cross membranes, therefore they are readily absorbable by biological systems, moreover NPs are highly reactive, especially because of the small volume compared to the big surface [2]. NPs and bacterial cells are not the only characters playing: NPs (which surface can be positive, negative or neutral) in a biological medium are able to attract biomolecules and ionic species dispersed in the milieu and to build a cover made of selected biomolecules, called protein corona $[12,13]$. It can be affirmed that it is very difficult to find NPs dispersed in a biological medium not surrounded by a protein corona [13]. NPs enveloped by the protein cover need to be considered as real new objects, in fact NPs will not react directly with the bacterial cells, but the protein corona will interact with the bacterial cell wall, plasma membrane and cytoskeletal structures (important for the internalization process) [12-16].

Analyzing TEM and STEM images in references 7, 10 and 11 it is possible to observe that NPs inside $S$. aureus are surrounded by membrane vesicles (MVs). This important information allows us to affirm that the uptake process occurs through endocytosis. During the endocytic process, xenobiotic material is internalized by a cell through the invagination of the plasma membrane and the scission of MVs that envelop the external material [17]. Figure 1A shows exactly these steps: we can see free NPs (not surrounded by a MV) out of the cell, and MVs enveloping PU NPs within the bacterium; furthermore details of the membrane lysis and a ruffle are visible as well $[7,10,11]$.

The NPs pathway from outside to inside the cell, delineates possible bacterial cytoskeletal structures. TEM and STEM images in references 7, 10 and 11 show that MVs loaded with one or more PU NPs are set out along linear routes that run across the whole cell, nearly drawing out the proof of the actual existence of the bacterial cytoskeleton [18-23].

Once a MV loaded with PU NPs has entered the bacterial cell, depending on the spatial distribution it can assume several names (early endosome, late endosome, lysosome and exosome) [24]. The last one (exosome) raises debates between authors since some accept that vesicular trafficking is possible only from outside the cell towards the inside [25], instead others agree that the trafficking can occur in both senses [26]. Our images finally put a stop to this argument, in fact in Figure 1 are visible MVs along linear pathways and in particular are shown MVs astride the bacterial cell and a MV outside the cell, in the external environment. This result confirms that the uptake process is reversible, vesicles can be secreted by the bacterium and suggests that the cytoskeleton plays a role in the secretion of MVs [27].

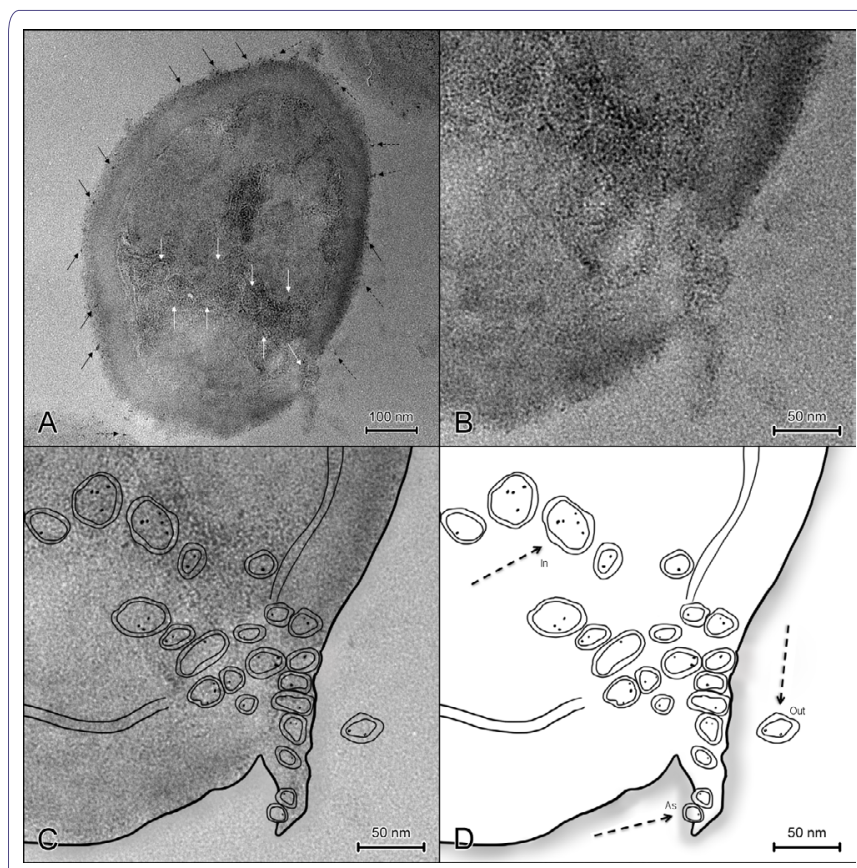

Figure 1: MVs loaded with NPs detectable in different regions.

A) TEM image of $S$. aureus after incubation with PU. PU particles can be observed on the cell wall (black $\uparrow$ ), inside the cell surrounded by membranes (white $\uparrow$ ) and in the external environment, in the proximity of the cell wall (black dashed $\uparrow$ ); B) Portion of Figure 1A (TEM image); C) In order to help the reader in the identification of the objects in Figure $1 \mathrm{~B}$, here it is presented the superimposition of the Figure 1B with its elaborated line art (Figure 1D); D) Line art of Figure 1B.

Details of the membrane lysis and a ruffle are visible. MVs with size ranging from $20 \mathrm{~nm}$ to $40 \mathrm{~nm}$ are set out along linear routes that link the ruffle to the cellular inner parts, drawing out the position of the cytoskeletal structures. MVs are loaded with PU NPs and each MV has a different dose of NPs within itself (one or more NPs).

The ruffle region, where it is visible the membrane lysis, is important not only for the endocytosis of PU NPs, but even for the secretion of MVs loaded with NPs. In fact in Figure 1, it is possible to observe MVs inside the bacterium (In), MVs astride the bacterial membrane (As) and a MV outside the cell (Out), in the extracellular medium.

This information confirms that vesicular trafficking occurs from inside the cell to the outside and vice versa, and suggests the involvement of the cytoskeletal structures not only in the uptake processes but even in the excreting ones.

Arrows in Figure 1D indicate MVs situated in three different regions that will be analyzed in Figure 2.

The secretion of MVs loaded with PU NPs from S. aureus supplies further proofs regarding the production of MVs from gram-positive bacteria. It was thought that the production of membranous structures was a peculiarity of gram-negative bacteria; instead it has been reviewed that MVs can be released by bacteria (both gram-negative and gram-positive), eukaryotes and even Archea [28]. The formation and secretion of extracellular vesicles, which can assume several names such as membrane vesicles (MVs), outer membrane vesicles (OMVs), exosomes, or shedding microvesicles, are therefore evolutionally conserved processes and the secreted vesicles preserve similar functions and mechanisms of release [28-31]. Lee et al. [32] demonstrated through electron microscopy images and proteomics characterization that gram-positive bacteria naturally produce MVs and excrete them in the extracellular milieu, strengthening the thesis that vesicular trafficking is a two-ways process. MVs derived from $S$. aureus are similar to the OMVs produced by gram-negative bacteria. OMVs are spherical vesicles 


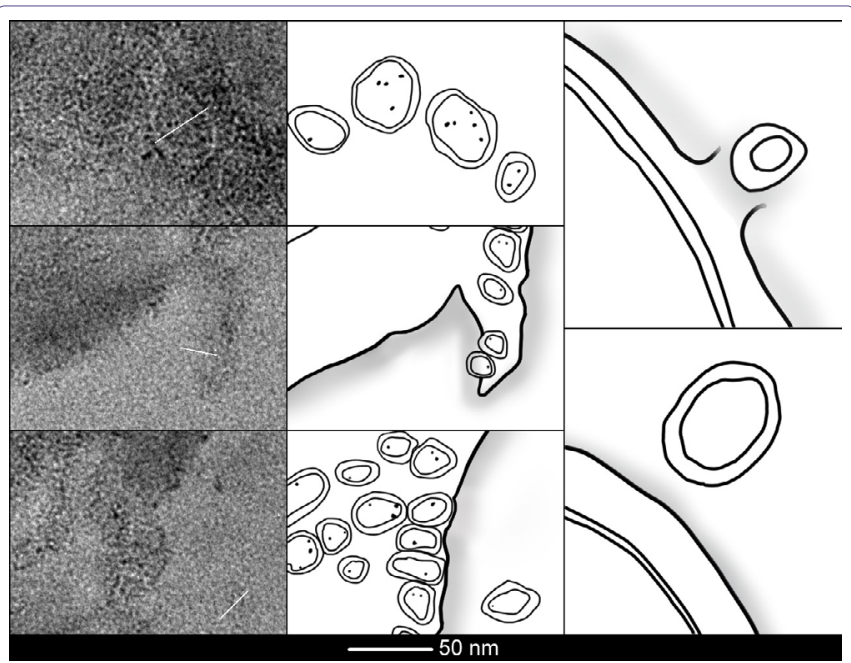

Figure 2: Role of Electron Microscopy in the identification of the morphology and spatial distribution of MVs loaded with PU NPs.

Three different portions of Figure $1 \mathrm{~A}$ that include MVs loaded with PU NPs inside the bacterial cell (In), astride the membrane (As) and outside the cell (Out) are shown in the first column and the respective line arts are visible in the central one.

In order to make a comparison between our outcomes and the results from literature, in the third column we present the line arts of Figures $1 \mathrm{~A}$ and $\mathrm{B}$ of Lee et al. [32]. The size of MVs (in the range of 20-40 nm) loaded with PU NPs is similar to that of the MVs shown by Lee et al. $(50 \mathrm{~nm})$ and in accordance with the literature $[31,32]$. The bacterial membrane lysis is visible both in Lee et al.'s line art and in our images, meaning that MVs represent a new strategy that bacteria have to excrete internal material (proteins, cellular components, virulence factors) as well as xenobiotic NPs.

with diameters ranging from $20 \mathrm{~nm}$ to $200 \mathrm{~nm}$; they are composed of lipopolysaccharides (LPS), phospholipids, outer membrane proteins, periplasmic proteins and are associated with several virulence factors (for instance toxins and adhesins). OMVs carry out different functions such as cell-cell communication (quorum sensing) [33], sensing of nutrients, killing of competitors, delivery of toxins or other virulence determinants to host cells, and modulation of host immune response [29,31,34-38]. The roles of MVs derived from gram-positive bacteria are similar to those of the OMVs: MVs are spherical vesicles smaller than the OMVs (MVs' diameter ranges from $20 \mathrm{~nm}$ to $100 \mathrm{~nm}$ ) that bacteria secrete in order to accomplish functions such as transfer of proteins, cell-cell signaling, sensing of nourishment, pathogenic purposes (infection), killing of competing bacteria [39], and antibiotic resistance [30-32].

In our experiment we face MVs loaded with xenobiotic material, PU NPs, derived from the biodestruction of dental prostheses carried out by $S$. aureus. It is worthwhile noting that NPs observed in our images cannot be ascribed to staining artifacts, since artifacts deriving for instance from lead citrate staining are larger and characterized by higher size dispersion.

We proceeded comparing the results obtained by our experiment with those showed by Lee et al. [32], in order to see if PU NPs could cause any modifications to the MVs. Three portions of the original image (Figure 1) that include MVs loaded with PU NPs inside the bacterial cell, astride the membrane and outside the cell are collected in Figure 2 (first column); these sections were elaborated and the resulting line arts are visible in the second column. In the third column it is presented the line art of Figures $1 \mathrm{~A}$ and B of Lee et al. [32]. The outcomes of the comparison pointed out that not all of our MVs bring the same amount of PU NPs, in fact MVs are loaded with one or more NPs; the size of MVs (20-40 nm) loaded with PU NPs is in accordance with the literature [31,32], the bacterial membrane lysis is visible both in Lee et al.s line art and in our images.

In addition we elaborated the inner MV (identified in Figure 2 with a white bar) with MATLAB ${ }^{\circ}$ in order to make the interpretation of the objects (membrane, NPs) easier (Figure 3); furthermore

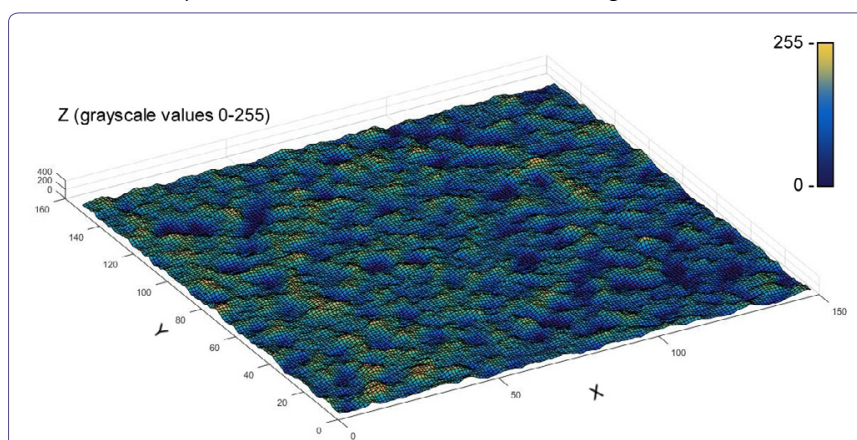

Figure 3: A different elaboration (via MATLAB ${ }^{\circledR}$ ) of the inner MV loaded with PU NPs inside the bacterial cell (In) discussed in Figures 2 and 4 is reported to help in the identification of the object's details.

The image is a surface plot which spatial coordinates are the $X$ and $Y$ axes the $Z$ axis refers to the original grayscale values, where the lower ones (associated to the blue color) correspond to the denser portions of the sample (membrane, NPs).

we made plots of the grayscale values of tiny sections of three types of MVs (In, As, Out) to highlight borders and inner material distribution (PU NPs) (Figure 4). The section of each MV analyzed is indicated in Figure 2 with a white bar.

The MVs formation and secretion from $S$. aureus occur both in vitro and in vivo [30,31], and increase the pathogenicity of $S$. aureus. In fact MVs represent a strategy for pathogenic bacteria to deliver a noxious cargo (included virulence factors) to host cells during infections $[30,31]$. S. aureus ability in evading the immune system and the developed high resistance against antibiotics can be linked again to the MVs' action [28,30,31]. MVs modulate host defense and response establishing a niche with host cells [34,38], indeed MVs could act as decoys for antibodies making the production of antibodies ineffective for the clearance of the intact organisms [28,40]; moreover active hydrolytic enzymes such as $\beta$-lactamases are secreted from bacteria via the MVs to target antimicrobial agents [30]. The interaction of MVs secreted by bacteria with host cells can be ambivalent, in fact it could afford a vantage for the host that recognizes a foreign invader, and for the microorganism itself that misdirects the immune system [28].

In our in vitro experiments staphylococcal MVs transport PU NPs outside the bacterium, in the extracellular medium. Transposing our results to an in vivo hypothetical situation, we would have an infection worsened by the delivery of NPs to target host human cells [41]. MVs represent a further access that PU NPs would have to reach lots of body areas, in fact MVs could enter in the systemic circle [40], and spread both the infection and the foreign material to several body districts. PU NPs could exit the bacterium not only through secreted MVs, but even as a consequence of bacterial death or through exocytosis. In the last case NPs would not be surrounded by a MV, and would be free in the host organism [41]. The characteristics of NPs make them highly reactive with biological systems, but nowadays further studies need to be done in order to understand the interactions that NPs have with bacterial cells and then with host cells, 

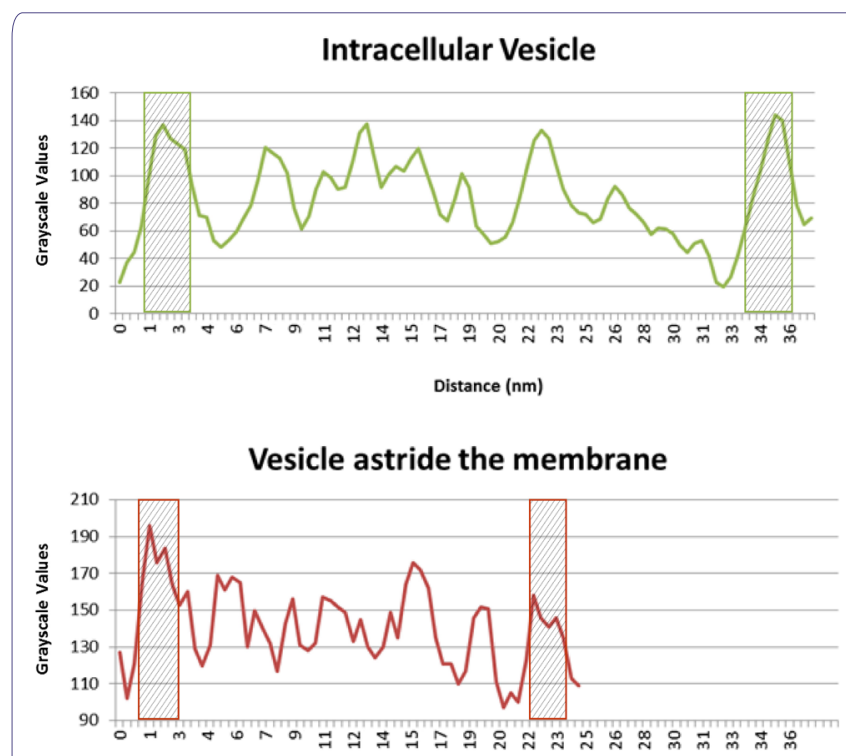

Distance $(\mathrm{nm})$

\section{Extracellular Vesicle}

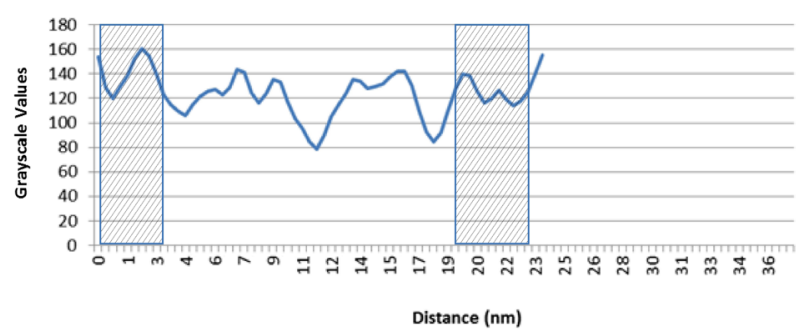

Figure 4: Plots of the grayscale values of tiny central sections of MVs detected inside the bacterial cell (In), astride the membrane (As) and outside the cell (Out).

The section of each MV analyzed is highlighted in Figure 2 with a white bar.

The $X$ axis represents the length (in $\mathrm{nm}$ ) of the section analyzed. The highlighted areas show the borders of the $\mathrm{MV}$, identified by the denser electron areas in the images (apart from those indicating the NPs and some bacterial inner structures). From this plot it is possible to deduce that: the vesicles' plasma membrane is 2-3 nm thick; the intracellular MV is bigger than the others $(35 \mathrm{~nm})$, whereas MVs astride the membrane and in the external medium are about $20 \mathrm{~nm}$ wide.

The $Y$ axis of the graphic represents the grayscale values. It goes from 0 (black) to 255 (white)

The gray values of the borders corresponding to the vesicles' plasma membrane of each MV are similar, proving that the object we are observing is the same (plasma membrane).

and to study the actual toxicity of nanosized materials and the levels of bioaccumulation within cells.

In this work we showed results carried out by an analysis of electron microscopy images. We would like to highlight the importance of electron microscopy in NPs-related studies. The details provided by electron microscopy images are not so "readily readable" [42], in fact beyond the first simply visible results there are the sharpness of the observation and the subtlety of the analysis.

With this study we described a new type of secretion pathway through which bacteria secrete proteins, cellular components, virulence factors, and a new kind of cargo (PU NPs). This work raises new issues about the toxicological risks and the dosimetry of NPs in bacterial MVs and in host cells. In the same time this study opens new ways to the nanomedicine in the comprehension of the mechanisms of the bacterial secretion of MVs and their interactions with host cells, and in the delivery of xenobiotic material guided by the dynamics of bacterial infections.

\section{References}

1. National Institute for Occupational Safety and Health (2012) Filling the Knowledge Gaps for Safe Nanotechnology in the Workplace. A Progress Report from the NIOSH Nanotechnology Research Center, 2004-2011. Department of Health and Human Services, Centers for Disease Control and Prevention. National Institute for Occupational Safety and Health, USA.

2. Revell PA (2006) The biological effects of nanoparticles. Nanotechnology Perceptions 2: 283-298.

3. Verma A, Stellacci $F(2010)$ Effect of surface properties on nanoparticle-cell interactions. Small 6: 12-21.

4. Krug HF, Wick $P$ (2011) Nanotoxicology: an interdisciplinary challenge. Angew Chem Int Ed Engl 50: 1260-1278.

5. Warheit DB (2008) How meaningful are the results of nanotoxicity studies in the absence of adequate material characterization? Toxicol Sci 101: 183-185.

6. Warheit DB (2010) Debunking some misconceptions about nanotoxicology. Nano Lett 10: 4777-4782.

7. Didenko LV, Avtandilov GA, Shevlyagina NV, Smirnova TA, Lebedenko IY, et al. (2012) Biodestruction of polyurethane by Staphylococcus aureus (an investigation by SEM, TEM and FIB). In: Mendez-Vilas A (ed.). Current microscopy contributions to advances in science and technology (Vol 1). Formatex Research Center, Spain. Pg no: 323-334.

8. Amara AA, Moawad H (2011) PHAC Synthases and PHA Depolymerases: The Enzymes that Produce and Degrade Plastic. IIUM Engineering Journal 12: 21-37.

9. Lenz RW, Marchessault RH (2005) Bacterial polyesters: biosynthesis, biodegradable plastics and biotechnology. Biomacromolecules 6: 1-8.

10. Didenko LV, Avtandilov GA, Shevlyagina NV, Shustrova NM, Smirnova TA, et al. (2013) Nanoparticles Production and Inclusion in S. aureus Incubated with Polyurethane: An Electron Microscopy Analysis. Open Journal of Medical Imaging 3: 69-73.

11. Curia R, Milani M, Didenko LV, Avtandilov GA, Shevlyagina NV, et al. (2014) Beyond the biodestruction of polyurethane: $S$. aureus uptake of nanoparticles is a challenge for toxicology. In: Mendez-Vilas A (ed.). Microscopy: advances in scientific research and education (Vol 1). Formatex Research Center, Spain. Pg no: 16-23.

12. Docter D, Distler U, Storck W, Kuharev J, Wünsch D, et al. (2014) Quantitative profiling of the protein coronas that form around nanoparticles. Nat Protoc 9: 2030-2044.

13. Xia XR, Monteiro-Riviere NA, Riviere JE (2010) An index for characterization of nanomaterials in biological systems. Nat Nanotechnol 5: 671-675.

14. Lundqvist M, Stigler J, Elia G, Lynch I, Cedervall T, et al. (2008) Nanoparticle size and surface properties determine the protein corona with possible implications for biological impacts. Proc Natl Acad Sci USA 105: 14265-14270.

15. Lynch I, Dawson KA (2008) Protein-nanoparticle interactions. Nanotoday 3: 40-47.

16. Salvati A, Pitek AS, Monopoli MP, Prapainop K, Baldelli FB, et al. (2013) Transferrin-functionalized nanoparticles lose their targeting capabilities when a biomolecule corona adsorbs on the surface. Nature Nanotechnology 8: 137-143.

17. Doherty GJ, McMahon HT (2009) Mechanisms of endocytosis. Annu Rev Biochem 78: 857-902.

18. Didenko LV, Shevlyagina NV, Curia R, Erega A, Milani M (2014) Electron Microscopy Furthers the Investigation of Bacteria-Nanoparticles Interactions Subcellular Dynamics. Global Journal of Medical Research: D Radiology, Diagnostic Imaging and Instrumentation 14: 13-23. 
19. Erickson HP (2001) Cytoskeleton. Evolution in bacteria. Nature 413: 30.

20. Graumann PL (2007) Cytoskeletal elements in bacteria. Annu Rev Microbiol 61: $589-618$

21. Mayer F (2006) Cytoskeletal elements in bacteria Mycoplasma pneumoniae, Thermoanaerobacterium sp., and Escherichia coli as revealed by electron microscopy. J Mol Microbiol Biotechnol 11: 228-243.

22. van den Ent F, Amos LA, Löwe J (2001) Prokaryotic origin of the actin cytoskeleton. Nature 413: 39-44.

23. Wickstead B, Gull K (2011) The evolution of the cytoskeleton. J Cell Biol 194: 513-525.

24. Denzer K, Kleijmeer MJ, Heijnen HF, Stoorvogel W, Geuze HJ (2000) Exosome: from internal vesicle of the multivesicular body to intercellular signaling device. J Cell Sci 19: 3365-3374.

25. Salvati A, Aberg C, dos Santos T, Varela J, Pinto P, et al. (2011) Experimental and theoretical comparison of intracellular import of polymeric nanoparticles and small molecules: toward models of uptake kinetics. Nanomedicine 7: 818-826.

26. Dombu CY, Kroubi M, Zibouche R, Matran R, Betbeder D (2010) Characterization of endocytosis and exocytosis of cationic nanoparticles in airway epithelium cells. Nanotechnology 21: 355102.

27. Mayer F, Gottschalk G (2003) The bacterial cytoskeleton and its putative role in membrane vesicle formation observed in a Gram-positive bacterium producing starch-degrading enzymes. J Mol Microbiol Biotechnol 6: 127-132.

28. Deatherage BL, Cookson BT (2012) Membrane vesicle release in bacteria eukaryotes, and archaea: a conserved yet underappreciated aspect of microbial life. Infect Immun 80: 1948-1957.

29. Beveridge TJ (1999) Structures of gram-negative cell walls and their derived membrane vesicles. J Bacteriol 181: 4725-4733.

30. Gurung M, Moon DC, Choi CW, Lee JH, Bae YC, et al. (2011) Staphylococcus aureus produces membrane-derived vesicles that induce host cell death. PLoS One 6: 27958.
31. Lee JC (2012) Staphylococcus aureus Membrane Vesicles and Its Potential Role in Bacterial Pathogenesis. Journal of Bacteriology and Virology 42: 181 188

32. Lee EY, Choi DY, Kim DK, Kim JW, Park JO, et al. (2009) Gram-positive bacteria produce membrane vesicles: proteomics-based characterization of Staphylococcus aureus-derived membrane vesicles. Proteomics 9: 54255436.

33. Mashburn LM, Whiteley M (2005) Membrane vesicles traffic signals and facilitate group activities in a prokaryote. Nature 437: 422-425.

34. Ellis TN, Kuehn MJ (2010) Virulence and immunomodulatory roles of bacterial outer membrane vesicles. Microbiol Mol Biol Rev 74: 81-94.

35. Kadurugamuwa JL, Beveridge TJ (1995) Virulence factors are released from Pseudomonas aeruginosa in association with membrane vesicles during normal growth and exposure to gentamicin: a novel mechanism of enzyme secretion. J Bacteriol 177: 3998-4008.

36. Kadurugamuwa JL, Beveridge TJ (1996) Bacteriolytic effect of membrane vesicles from Pseudomonas aeruginosa on other bacteria including pathogens: conceptually new antibiotics. J Bacteriol 178: 2767-2774.

37. Kadurugamuwa JL, Beveridge TJ (1997) Natural release of virulence factors in membrane vesicles by Pseudomonas aeruginosa and the effect of aminoglycoside antibiotics on their release. J Antimicrob Chemother 40: 615-621.

38. Kuehn MJ, Kesty NC (2005) Bacterial outer membrane vesicles and the host-pathogen interaction. Genes Dev 19: 2645-2655.

39. Li Z, Clarke AJ, Beveridge TJ (1998) Gram-negative bacteria produce membrane vesicles which are capable of killing other bacteria. J Bacteriol 180: 5478-5483.

40. Olsen I, Amano A2 (2015) Outer membrane vesicles - offensive weapons or good Samaritans? J Oral Microbiol 7: 27468.

41. Curia R, Milani M, Didenko LV, Shevlyagina NV (2013) Electron microscopy broadens the horizons of toxicology: The role of nanoparticles vehiculated by bacteria. Current Topics in Toxicology 9: 93-98.

42. Postek MT, Vladár AE (2013) Does your SEM really tell the truth?--How would you know? Part 1. Scanning 35: 355-361. 